\title{
Seat Vibration for Heart Monitoring in a Moving Automobile*
}

\author{
Amelie Bonde \\ Carnegie Mellon University \\ Moffett Field, California \\ amelie@cmu.edu \\ Shijia Pan \\ Carnegie Mellon University \\ Pittsburgh, PA \\ shijiapan@cmu.edu
}

\author{
Extended Abstract
}

\author{
Mostafa Mirshekari \\ Carnegie Mellon University \\ Pittsburgh, PA \\ mmirshekari@cmu.edu \\ Hae Young Noh \\ Carnegie Mellon University \\ Pittsburgh, PA \\ noh@cmu.edu
}

\author{
Jonathon Fagert \\ Carnegie Mellon University \\ Pittsburgh, PA \\ jfagert@andrew.cmu.edu \\ Pei Zhang \\ Carnegie Mellon University \\ Moffett Field, California \\ peizhang@cmu.edu
}

\begin{abstract}
Continuous heart rate monitoring in cars can allow ambient health monitoring and help track driver stress and fatigue. We present a data set from accelerometers embedded in a car seat which includes ten people sitting in the passenger seat of a moving car, and surface ECG data of each user to provide ground truth of the heartbeat. This data can be used to analyze the heart activity of people in cars, despite the presence of high levels of noise from car motion, human motion, and the car engine.
\end{abstract}

\section{CCS CONCEPTS}

- Human-centered computing $\rightarrow$ Ubiquitous and mobile computing systems and tools; Ubiquitous and mobile computing design and evaluation methods;

\section{ACM Reference Format:}

Amelie Bonde, Mostafa Mirshekari, Jonathon Fagert, Shijia Pan, Hae Young Noh, and Pei Zhang. 2018. Seat Vibration for Heart Monitoring in a Moving Automobile: Extended Abstract. In The 1st Workshop on Data Acquisition To Analysis (DATA'18), November 4, 2018, Shenzhen, China. ACM, New York, NY, USA, 2 pages. https://doi.org/10.1145/3277868.3277872

\section{INTRODUCTION}

Continuous heart rate and heart rate variability (HRV) monitoring in cars can allow for ambient health monitoring. Short-term HRV monitoring can be used to track stress and fatigue, which are important driver safety issues [2,11].

Prior work takes a number of approaches including photoplethysmography, electrocardiography (ECG), and cameras $[4,6,9]$. These methods can be useful for heart-rate monitoring in static environments but are inconvenient to the user or inaccurate for HRV measurements. In particular, these approaches fail for high-noise

\footnotetext{
*Produces the permission block, and copyright information

Permission to make digital or hard copies of all or part of this work for personal or classroom use is granted without fee provided that copies are not made or distributed for profit or commercial advantage and that copies bear this notice and the full citation on the first page. Copyrights for components of this work owned by others than ACM must be honored. Abstracting with credit is permitted. To copy otherwise, or republish, to post on servers or to redistribute to lists, requires prior specific permission and/or a fee. Request permissions from permissions@acm.org.

DATA'18, November 4, 2018, Shenzhen, China

(C) 2018 Association for Computing Machinery.

ACM ISBN 978-1-4503-6049-4/18/11.

https://doi.org/10.1145/3277868.3277872
}

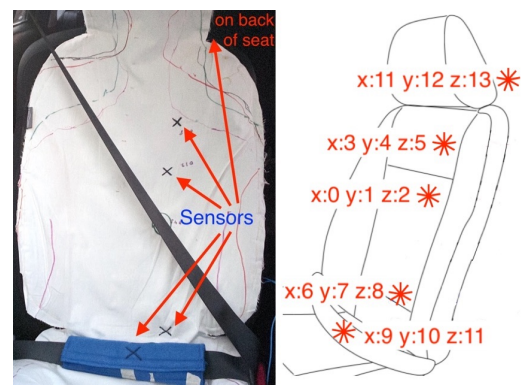

Figure 1: A hardware setup photo and a sensor layout diagram shows the channel label for each sensor axis.

in-car environments subject to periodic noise such as car engine vibration and impulsive noise from subject movement.

We propose using accelerometers embedded in a car seat to measure the motion of the heart. Vibration sensing such as that from accelerometers has been successful in scenarios such as identification [7], localization [5], and health tracking [3]. We present a data set of accelerometer data from ten people sitting in the passenger seat of a car. We use multiple sensors embedded in the car seat to account for variations in body size and position. The experiments are conducted based on guidelines approved by the CMU Institutional Review Board (IRB) review (Registration Number: IRB00000666).

\section{EXPERIMENTS}

To evaluate the application of vibration for heart rate monitoring, we performed a set of experiments in a car. The make, model and year of the car we used was Mazda CX5, 2016. We use the W354C03_010G10 piezoelectric accelerometer, sampled at $10 \mathrm{kHz}$ [8]. We put the sensors on the passenger seat because passengers move more freely and would give us more varied motion noise. To get the ground truth [10] for heart rate, the subjects wear a Zephyr Bioharness belt (capacitive ECG). In this section, we first describe the number and location of the sensors (in Section 2.1). Then, we discuss the human subjects details (in Section 2.2). Finally, we discuss the file settings and format for the dataset (in Section 2.3).

\subsection{Sensor Placement}

The sensors were embedded into a cloth pad that was attached to the passenger seat of a car. A photo of the cloth pad on the seat is 
Table 1: Subject Experiment Details

\begin{tabular}{|c|c|c|c|}
\hline Subject & Gender & Height & Car Status \\
\hline Subject 1 & Male & 5’11" & Idle \\
\hline Subject 2 & Male & 5’10” & Idle \\
\hline Subject 3 & Male & $5 ’ 11^{\prime \prime}$ & Idle \\
\hline Subject 4 & Female & $5 ’ 3 "$ & Idle \\
\hline Subject 5 & Female & $5 ’ 0 "$ & Moving \\
\hline Subject 6 & Female & $5 ' 6^{\prime \prime}$ & Moving \\
\hline Subject 7 & Female & 4’10" & Moving \\
\hline Subject 8 & Male & $63^{\prime \prime}$ & Moving \\
\hline Subject 9 & Male & 6’0" & Moving \\
\hline Subject 10 & Female & $5^{\prime} 5^{\prime \prime}$ & Moving \\
\hline
\end{tabular}

shown in Figure 1a. We have utilized five sensors including three sensors on the backrest at different heights, one sensor on the seat belt across the lap, and one sensor on the back of the headrest. The top two sensors on the backrest accommodate different heights of drivers and passengers and are located slightly to one side of the backrest in order to be closer to the heart. The sensor at the bottom of the backrest is used to characterize noise from the person talking or moving as well as noise from car motion and the engine and hence, is put further from the heart. The sensor on the seat belt is used to pick up the heartbeat through the stomach when the person leans forward. Finally, in order to characterize noise from the car, we have utilized the sensor on the back of the headrest. The dataset includes the vibration from three axes for five sensors and therefore is formed into 15 columns of data. Figure $1 \mathrm{~b}$ shows the labeled column for each axis of data, starting from column 0 .

\subsection{Subject Details}

In this data set we have included vibration data from 10 subjects, 5 women and 5 men. Table 1 shows the gender, height, and whether the car was moving for each subject. For the first four subjects, we let the car sit in the parking lot with the engine running, while for the last 6 subjects, we drove around the neighborhood of Moffett Field, CA stopping at stop signs and getting on and off the nearby highway. Our idling subject data was analyzed in some of our previous work [1], but the moving car data has yet to be published.

\subsection{Data File Settings}

The data was recorded in comma-separated-value (CSV) text files, each of which contains a 23-line header and then 100,000 lines of data in 16 columns, comprising 10 seconds of data. The first column contains the timestamp and the next 15 columns contain the 3 axes of the 5 sensors in order of axis, so $x$, then $y$, then $z$ axis data. We expect the $z$ axis data to be the most useful because it is parallel to the direction the heart beats. Figure 2 presents an example usage of the dataset. We have plotted 3 seconds of the z-axis data of a single sensor from Subject 2 (channel 2 from file "data_16-09-16_1632_049.csv") in Figure 2, with the locations of the heartbeats outlined in boxes.

The ground truth for each experiment is collected in a separate CSV file, as capacitive ECG data with a sampling rate of $250 \mathrm{~Hz}$. In this file, there are two columns of data, the first column is the timestamp and the second column is the ECG signal. The ECG signal suffers from motion artifacts due to the motion of the subject

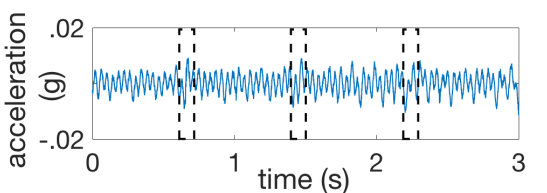

Figure 2: 3 seconds of raw $\mathrm{z}$-axis data from a subject.

and in some cases, an ill-fitting chest harness for the ECG. Using a high-pass filter on this data is recommended in order to reduce motion artifacts in the ECG data.

\section{CONCLUSION}

To evaluate the application of vibration for heart rate monitoring in a car, we performed a series of experiments. We presented a set of data from ten people sitting in a car that has multiple accelerometers embedded in the seat. We hope the research community finds value in analyzing this data for in-vehicle heart-rate monitoring.

\section{ACKNOWLEDGEMENTS}

This research was supported in part by the National Science Foundation Graduate Research Fellowship Program (under Grant No. DGE 1745016), Carnegie Mellon UniversityâĂŹs Mobility21 National University Transportation Center (grant number 69A3551747111), Intel, Google and AiFi. The views and conclusions contained here are those of the authors and should not be interpreted as necessarily representing the official policies or endorsements, either express or implied, of Carnegie Mellon University, the National Science Foundation, or the U.S. Government or any of its agencies.

\section{REFERENCES}

[1] Amelie Bonde, Shijia Pan, Zhenhua Jia, Yanyong Zhang, Hae Young Noh, and Pei Zhang. 2018. VVRRM: Vehicular Vibration-Based Heart RR-Interval Monitoring System. In Proceedings of the 19th International Workshop on Mobile Computing Systems \& Applications. ACM, 37-42.

[2] Niels Egelund. 1982. Spectral analysis of heart rate variability as an indicator of driver fatigue. Ergonomics 25, 7 (1982), 663-672.

[3] Zhenhua Jia, Amelie Bonde, Sugang Li, Chenren Xu, Jingxian Wang, Yanyong Zhang, Richard E Howard, and Pei Zhang. 2017. Monitoring a Person's Heart Rate and Respiratory Rate on a Shared Bed Using Geophones. In Proceedings of the 15th ACM Conference on Embedded Network Sensor Systems. ACM, 6.

[4] Boon-Giin Lee and Wan-Young Chung. 2012. A smartphone-based driver safety monitoring system using data fusion. Sensors 12, 12 (2012), 17536-17552.

[5] M. Mirshekari, S. Pan, J. Fagert, E.M. Schooler, P. Zhang, and H.Y. Noh. 2018. Occupant localization using footstep-induced structural vibration. Mechanical Systems and Signal Processing 112 (2018), 77-97.

[6] Nermine Munla, Mohamad Khalil, Ahmad Shahin, and Azzam Mourad. 2015. Driver stress level detection using HRV analysis. In Advances in Biomedical Engineering (ICABME), 2015 International Conference on. IEEE, 61-64.

[7] S. Pan, T. Yu, M. Mirshekari, J. Fagert, A. Bonde, O. J. Mengshoel, and P. Zhang. 2017. FootprintID: Indoor Pedestrian Identification through Ambient Structural Vibration Sensing. Proceedings of the ACM on Interactive, Mobile, Wearable and Ubiquitous Technologies 1, 3 (2017), 89.

[8] PCB Piezotronics. [n. d.]. W354C03_010G10 datasheet. http://www.pcb.com/ Products.aspx?m=W354C03 010G10.

[9] Ye Sun and Xiong Bill Yu. 2014. An innovative nonintrusive driver assistance system for vital signal monitoring. IEEE journal of biomedical and health informatics 18, 6 (2014), 1932-1939.

[10] Zephyr Performance Systems. [n. d.]. Zephyr BioModule Device. https://www. zephyranywhere.com/benefits/physiological-biomechanical.

[11] Adrian H Taylor and Lisa Dorn. 2006. Stress, fatigue, health, and risk of road traffic accidents among professional drivers: the contribution of physical inactivity. Annu. Rev. Public Health 27 (2006), 371-391. 